\title{
Brauchen Kinder funktionelle Lebensmittel?
}

\author{
Das Angebot an funktionellen \\ Lebensmitteln wird immer \\ größer. Wie steht es, insbeson- \\ dere bei Kindern, um Nutzen \\ und Risiken? Wir fragten Prof. \\ Dr. troph. Mathilde Kersting \\ vom Forschungsinstitut für \\ Kinderernährung (FKE) in \\ Dortmund.
}

MMW: Was ist ein funktionelles Lebensmittel?

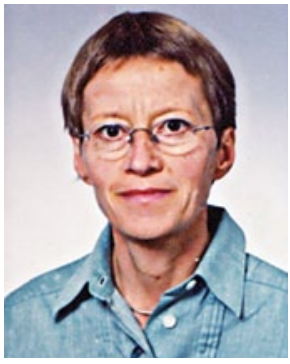

„Vorteilhaft, sich auf herkömmliche Lebensmittel zu konzentrieren."
Kersting: Funktionelle Lebensmittel i. e. S. enthalten Zusätze, die über die reine Nährstoffwirkung hinaus gesundheitsfördernde Effekte haben sollen, z. B. kognitive Leitungssteigerung oder Infektabwehr. In Säuglingsmilch werden Probiotika oder/ und Präbiotika sowie Omega-3-Fettsäuren zugesetzt. Pro- und Präbiotika sollen z. B. Allergien und Infektionen vorbeugen. Studien haben allerdings zu widersprüchlichen Ergebnissen geführt. Omega-3-Fettsäuren sollen die kognitive und visuelle Leistung verbessern. Langfristige positive Effekte sind bisher nicht eindeutig nachgewiesen worden, allerdings auch keine Nachteile.
MMW: Gibt es funktionelle Lebensmittel, die bei Kindern wirklich einen Nutzen haben?

Kersting: In Deutschland ist Jodmangel nach wie vor weit verbreitet. Jodmangel kann die Entwicklung von Kindern nachhaltig beeinträchtigen. Jodiertes Speisesalz kann daher als funktionelles Lebensmittel betrachtet werden, das für die Entwicklung von Kindern besonders wichtig ist.

MMW: Was ist von Vitamintabletten zu halten?

Kersting: Es gibt eine neue Empfehlung der Ernährungskommission der Deutschen Gesellschaft für Kinder- und Jugendmedizin zur Vitamin-D-Supplementierung. Es hat sich herausgestellt, dass bei Kindern die Versorgung mit Vitamin D ungenügend ist. Zur Unterstützung der Knochengesundheit sollten sie deswegen täglich eine Tablette mit $400 \mu \mathrm{g}$ Vitamin D erhalten.
MMW: Können von funktionellen Lebensmitteln auch Risiken ausgehen?

Kersting: Man hat z. B. herausgefunden, dass die Supplementierung mit Karotinoiden bei Rauchern das Lungenkarzinomrisiko erhöht. Bei Kindern gibt es keine Studien zu möglichen Langzeitrisiken.

MMW: Bieten funktionelle Lebensmittel irgendeinen Vorteil gegenüber einer gesunden Mischkost?

Kersting: Nein. Mit Ausnahme von Jodsalz und der Vitamin-D-Supplementierung gibt es derzeit keine Notwendigkeit für die Anreicherung von Lebensmitteln mit Nährstoffen oder anderen Substanzen mit potenziell gesundheitsförderlichen Wirkungen. Es hat sogar Vorteile, sich auf die herkömmlichen Lebensmittel zu konzentrieren, weil die auch Mischungen von Substanzen enthalten, deren Einzelwirkung wir gar nicht kennen. Obst und Gemüse enthalten z. B. Vitamine, sekundäre Pflanzenstoffe und Ballaststoffe. Oft lässt sich gar nicht trennen, auf welche Komponenten die gesundheitsförderlichen Effekte zurückgehen. - INTERVIEW: Dr. BeATE SCHUMACHER

\section{SYMPTOME RICHTIG DEUTEN}

\section{Wie krank macht der Zahndurchbruch?}

- Wenn die ersten Zähne kommen, sind manche Kinder stark beeinträchtigt. Aber welche Symptome dürfen wirklich dem Zahndurchbruch zugeschrieben werden - und welche nicht? Kinderzahnärzte aus Brasilien haben acht Monate lang den Gesundheitszustand von 47 zahnenden Kleinkindern dokumentiert. Die Kinder im Alter zwischen 5 und 15 Monaten, von denen keines an einer chronischen Erkrankung litt, bekamen insgesamt 231 Zähne. Der Zahndurchbruch war verbunden mit einem signifikanten Anstieg der Körpertemperatur. Im Mittel lagen die Werte allerdings nur $0,12^{\circ} \mathrm{C}$ (aurikulär) bzw. $0,01^{\circ} \mathrm{C}$ (axillar) höher als sonst. Die maximal gemessene Temperatur betrug $36,8{ }^{\circ} \mathrm{C}$ bzw. $36,6{ }^{\circ} \mathrm{C}$. Außerdem trat eine Reihe von Symptomen signifikant häufiger am Tag des Zahndurchbruchs und am Folgetag auf.
An erster Stelle waren das: Reizbarkeit, vermehrter Speichelfluss, eine laufende Nase und Appetitlosigkeit. Etwas weniger häufig waren Schlafstörungen, Hautausschlag und Diarrhö. Kein Kind litt an Krämpfen oder Koliken. Nur in seltenen Fällen wurde über Erbrechen berichtet. Fieber oder andere schwere Krankheitszeichen gehören nach dieser Studie also nicht zu den typischen Symptomen zahnender Kinder. Das Fazit der Autoren lautet daher: "Wenn Kinder gravierende Befunde und Beschwerden haben, sollte man nicht vorschnell das Zahnen dafür verantwortlich machen, sondern zuerst nach anderen Ursachen suchen."

Ramos-Jorge J et al. Pediatrics, published online August 8, 2011

Die ersten Zähnchen machen quengelig, aber nicht richtig krank. 